\title{
Whole-genome sequencing reveals the mechanisms for evolution of streptomycin resistance in Lactobacillus plantarum
}

\author{
Fuxin Zhang, ${ }^{*}$ Jiayuan Gao, ${ }^{*}$ Bini Wang, ${ }^{*}$ Dongxue Huo, $†$ Zhaoxia Wang, $\ddagger$ Jiachao Zhang, $\dagger^{1}$ and Yuyu Shao*1 \\ ${ }^{*}$ College of Food Engineering and Nutritional Science, Shaanxi Normal University, Xi'an 710119, Shaanxi, P. R. China \\ †College of Food Science and Technology, Hainan University, Haikou 570228, Hainan, P. R. China \\ †College of Food Science and Engineering, Inner Mongolia Agricultural University, Hohhot 010018, Inner Mongolia, P. R. China
}

\section{ABSTRACT}

In this research, we investigated the evolution of streptomycin resistance in Lactobacillus plantarum ATCC14917, which was passaged in medium containing a gradually increasing concentration of streptomycin. After $25 \mathrm{~d}$, the minimum inhibitory concentration (MIC) of L. plantarum ATCC14917 had reached $131,072 \mu \mathrm{g} / \mathrm{mL}$, which was 8,192-fold higher than the MIC of the original parent isolate. The highly resistant L. plantarum ATCC14917 isolate was then passaged in antibiotic-free medium to determine the stability of resistance. The MIC value of the L. plantarum ATCC14917 isolate decreased to $2,048 \mu \mathrm{g} / \mathrm{mL}$ after $35 \mathrm{~d}$ but remained constant thereafter, indicating that resistance was irreversible even in the absence of selection pressure. Whole-genome sequencing of parent isolates, control isolates, and isolates following passage was used to study the resistance mechanism of L. plantarum ATCC14917 to streptomycin and adaptation in the presence and absence of selection pressure. Five mutated genes (single nucleotide polymorphisms and structural variants) were verified in highly resistant $L$. plantarum ATCC14917 isolates, which were related to ribosomal protein S12, LPXTG-motif cell wall anchor domain protein, LrgA family protein, Ser/Thr phosphatase family protein, and a hypothetical protein that may correlate with resistance to streptomycin. After passage in streptomycin-free medium, only the mutant gene encoding ribosomal protein S12 remained; the other 4 mutant genes had reverted to the wild type as found in the parent isolate. Although the MIC value of L. plantarum ATCC14917 was reduced in the absence of selection pressure, it remained 128-fold higher than the MIC value of the parent isolate, indicating that ribosomal protein S12 may play an important role in streptomycin resistance. Using the mobile elements

Received June 12, 2017.

Accepted December 13, 2017.

${ }^{1}$ Corresponding authors: zhjch321123@163.com and icheesemaking@yahoo.com database, we demonstrated that streptomycin resistance-related genes in L. plantarum ATCC14917 were not located on mobile elements. This research offers a way of combining laboratory evolution techniques and whole-genome sequencing for evaluating antibiotic resistance in probiotics.

Key words: Lactobacillus plantarum, antibiotic resistance, minimum inhibitory concentration, experimental evolution, whole-genome sequencing

\section{INTRODUCTION}

Probiotics are live cultures of microbes that, when consumed in food or supplements, alter the intestinal microbial balance resulting in improved health and growth (Sánchez et al., 2017). Probiotics used in this way are generally recognized as safe (GRAS; Monahan, 2011). However, the Food and Agriculture Organization of the United Nations and the World Health Organization have produced guidelines for the safety evaluation of probiotics, which must be followed before any probiotic can be used commercially in food, including assessments for antibiotic resistance (FAO-WHO, 2002).

Globally, antimicrobial resistance is a growing problem that poses a threat to human and animal health (Laxminarayan et al., 2016). With wider use of probiotics, their antibiotic resistance status requires attention. Currently, relatively few standard methods exist for the evaluation of antibiotic resistance in probiotics. Although protocols are available for detection of antibiotic resistance in lactic acid bacteria, standards are scarce in antibiotic resistance gene assessment. Protocols include Clinical and Laboratory Standards Institute (CLSI) M45-P antimicrobial dilution disk susceptibility testing, which involves determining the MIC of a few genera of lactic acid bacteria and antibiotics (CLSI, 2006). The comprehensive standards of International Organization for Standardization (ISO) and the International Dairy Federation (IDF) include norms for the MIC detection methods for lactic acid bacteria (ISO10932/IDF223) that define all the parameters, 
including the medium to use, cultivation conditions, isolates to include for quality control, and so on (ISOIDF, 2010). Furthermore, EFSA (2012) defines cut-off values for resistance in lactic acid bacteria to different antibiotics. When the MIC of the probiotic is higher than the defined breakpoint value, then any antibioticresistant gene present should be identified before the probiotic can be used safely in foods. However, there are no standards for the detection and identification of antibiotic resistance genes or their transferability between different isolates and species. This is because resistance genes are numerous, making it difficult to standardize methods, and their detection requires reference isolates and gene sequences, which are few or incomplete.

The potential to use combinations of probiotics and antibiotics is a curative strategy (Govender et al., 2016), but it remains controversial. Probiotics with low-level resistance may not be effective in combination with antibiotics if they are inhibited by the antibiotics, whereas probiotics with high-level resistance may represent a safety issue due to the potential for transfer of antibiotic resistance genes from the probiotic to cooccurring pathogens. Research is required to solve the therapeutic issues that currently limit the combined use of antibiotics and probiotics. In particular, more information is required on the identity and frequency of antibiotic resistance genes in probiotics with high-level resistance and the likelihood that these genes might transfer to other species.

According to current standards (EFSA, 2012), probiotics in foods should be susceptible to antibiotics. Therefore, experimental laboratory evolution studies are the most effective way to obtain isolates with high-level resistance and to study bacterial adaptation trajectory in the environment (Lukačišinová and Bollenbach, 2017). Bacteria can be grown experimentally under continuous antibiotic pressure to encourage the selection of resistant genotypes adapted to the environment. In such experiments, one or more influencing factors can be established and applied continuously or intermittently. By detecting phenotypic changes over time, the influence of particular variables on the adaptation process can be studied over evolutionary time. In such a highly controlled laboratory experiment, other variables can be minimized effectively. Experimentally passaged isolates can be sampled at different time points and compared with the original isolate grown in the absence of selection pressure to study the relationship between genotype and their regulated phenotype. In this way, the potential mechanisms of adaptation in bacteria under selection pressure can be elucidated. These types of studies have been used widely to shed light on mechanisms of adaptation for antibiotic resistance in pathogenic bacteria under sustained drug selection (Baym et al., 2016; Cairns et al., 2017; Levin-Reisman et al., 2017). However, they have only rarely been used in the study of probiotics, especially antibiotic resistance adaptation in probiotics. A recent publication reported the antibiotic resistance and genomic changes of the probiotic strain Lactobacillus casei Zhang under long-term (10 mo) amoxicillin or gentamicin pressure, and showed that Lb. casei Zhang has high genome stability under antibiotic selection forces; this research is a good reference for study on genome adaptive evolution in Lactobacillus under longterm antibiotic stress (Wang et al., 2017).

Lactobacillus spp. are important probiotics and commonly found in dairy products, as well as in the gastrointestinal tract, where they contribute to the regulation and improvement of the intestinal flora of humans and promote the absorption of nutrients (Stefanovic et al., 2017). In our previous research, we selected for high streptomycin resistance in Lactobacillus plantarum IMAU60045 using a laboratory evolution approach; the MIC value was 1,024-fold higher in the adapted isolate than the original isolate. Using quantitative PCR on 2 streptomycin-related genes $[\operatorname{ant}(6)$ and $\operatorname{aadA}]$, we were able to make preliminary studies on the potential mechanism for adaptation but we failed to identify specific mutations at the whole-genome level (Shao et al., 2015).

Advances in next-generation sequencing technologies allow whole-genome sequencing and make it possible to trace the emergence of variants in a population and analyze genomic differences between individuals within a population under different selection pressures or environments (Barrick and Lenski, 2013; Tenaillon et al., 2016; Lenski, 2017). Based on the whole genome of a reference isolate, subsequent isolates developed under different stressful environments can be sequenced and their sequences compared with the original reference genome to identify mutations. In this study, we investigated streptomycin resistance mechanisms in a probiotic strain, L. plantarum ATCC14917 (Wang et al., 2016; Tomaro-Duchesneau et al., 2014). Isolates with high-level resistance were produced using laboratory evolution techniques under gradual and incrementally increasing concentrations of streptomycin. The adaptation and resistance mechanisms of L. plantarum ATCC14917 were further studied by whole-genome sequencing of the adapted isolates compared with the original isolate. Then, the mobile element database was used to analyze the potentially transferability of the mutant genes to determine the safety of isolates with high-level streptomycin resistance, which provides the method for evaluating antibiotic resistance in probiotics. 


\section{MATERIALS AND METHODS}

\section{Bacterial Isolate, Culture Media, and Growth Conditions}

Lactobacillus plantarum ATCC14917 was obtained from the American Type Culture Collection (ATCC, Manassas, VA). The complete genome sequence for $L$. plantarum ATCC14917 was already available in GenBank (accession number NZ_ACGZ00000000.2), providing the basis for data analysis of the whole-genome sequencing of adapted isolates from the laboratory evolution study.

The culture media and growth conditions were those described in the ISO10932/IDF223 standard (ISO-IDF, 2010). The isolate was activated in de Man, Rogosa and Sharpe (MRS) broth (M1175, Oxoid, Basingstoke, UK) at $28^{\circ} \mathrm{C}$ for $24 \mathrm{~h}$. For antimicrobial susceptibility tests and laboratory evolution, LAB (lactic acid bacteria) susceptibility medium (LSM) was used, which is a mixture of 90\% ISO-Sensitest broth (CM0473, Oxoid) and 10\% MRS broth (Klare et al., 2005).

\section{Antibiotic Susceptibility Test}

Streptomycin sulfate salt (Sigma-Aldrich, St. Louis, MO) was weighed out according to the potency of the active compound in streptomycin sulfate salt $(721 \mathrm{IU} /$ $\mathrm{mg}$ ), dissolved, and diluted with LSM broth to make streptomycin stock solutions $(131,072 \mu \mathrm{g} / \mathrm{mL})$, which were filter-sterilized $(0.22 \mu \mathrm{m})$ and stored at $-80^{\circ} \mathrm{C}$. Antibiotic susceptibility of L. plantarum ATCC14917 to streptomycin was determined using the microdilution method according to ISO-IDF (2010). All experiments were performed in triplicate.

\section{Experimental Evolution of L. plantarum ATCC14917 in a Streptomycin-Adverse Environment}

The experimental evolution procedure followed that of Lázár et al. (2014) and Shao et al. (2015). Briefly, 2 replicate colonies of $L$. plantarum ATCC14917 were activated on MRS agar. The initial streptomycin concentration in LSM broth was $8 \mu \mathrm{g} / \mathrm{mL}$ in the laboratory evolution study. This concentration was selected because it was sufficient to inhibit L. plantarum ATCC14917 population growth by $50 \%\left(\mathrm{IC}_{50}\right)$. For every passage (i.e., transferral by subculturing), $40 \mu \mathrm{L}$ of inoculum (1\%) from the previous subculture was added to 3.96 $\mathrm{mL}$ of streptomycin-LSM (LSM-Str) and cultivated at $28^{\circ} \mathrm{C}$ for $24 \mathrm{~h}$, by which time the culture had entered the stationary phase. Passage in streptomycin-free medium (LSM) was run as a control. During passage, the concentration of streptomycin was increased 1.5 times every $5 \mathrm{~d}$ (i.e., at every fifth transfer). The passage was halted when the streptomycin concentration had reached its upper solubility. During and after passage, samples were taken and preserved in glycerol solution at $-80^{\circ} \mathrm{C}$ for further analysis. The MIC against streptomycin during passage was determined for each lineage every $5 \mathrm{~d}$.

\section{Evolution of Resistant L. plantarum in the Absence of Antibiotic Pressure}

The high-level streptomycin-resistant L. plantarum ATCC14917 isolates produced were inoculated in LSM without antibiotic (i.e., no selection pressure) and passaged as described previously until the MIC values stabilized. Samples of isolates were taken during and after passage and preserved in glycerol solution at $-80^{\circ} \mathrm{C}$ for further analysis.

\section{Whole-Genome Sequencing, Bioinformatics Analysis, and Validation of Variants}

Genomic DNA from the original (ancestral) parent $L$. plantarum ATCC14917 isolate, the control isolate, and the passaged lineages (i.e., end point of passage) was extracted using a bacterial genomic DNA kit (DNeasy Blood \& Tissue Kit, Qiagen, Valencia, CA) according to the manufacturer's instructions. Genomic DNA concentrations were quantified by Qubit fluorometer (Thermo Fisher Scientific Inc., Waltham, MA); integrity and purity were analyzed by electrophoresis in $1 \%$ agarose gels at $100 \mathrm{~V}$ for $40 \mathrm{~min}$. High-quality DNA was used to construct 350-bp DNA libraries, which were prepared for sequencing on an Illumina HiSeq 4000 platform (Illumina Inc., San Diego, CA) with PE150 (pair-end 150-bp sequencing) strategies. Raw reads (in FASTQ format) were obtained by transformation of the fluorescence signal figures from high-throughput sequencing using Casava (v1.8.2; Illumina Inc.) base calling. Raw reads were then analyzed by CLC Genomics Workbench version 9.5.2 (Qiagen Inc., Germantown, MD), which contains a suite of tools for sequencing data quality control, trimming the sequences based on the quality, mapping reads to the reference sequence, removing the duplicate mapped reads due to the amplification process before sequencing, and detecting variants. Sequencing data analysis was performed and parameters for each step of analysis were set accordingly. The trim using quality scores' limit of 0.05 and maximum of 2 ambiguities was set for quality trimming. Mapping reads to the reference genome should reach at least $80 \%$ identity, and at least $50 \%$ alignment (length fraction of 0.5 ) must match the reference sequence; match score and mismatch score were set by default 
to 1 and 2, respectively. A maximum representation of minority sequence of 20 was set for duplicate removal. For variant detection, variants covered by minimum of 10 reads were called; minimum count and minimum frequency were set at 2 and 1\%, respectively. Variants of SNP, insertions and deletions (InDel), and structural variants (SV) were detected and validated by Sanger sequencing. The Kyoto Encyclopedia of Genes and Genomes database (KEGG; http://www.genome.jp/ kegg/) was used to study the metabolic pathways and annotate the functions of the mutated genes.

\section{Prediction of Mobility of Streptomycin Resistance Genes in Mutated Lineages}

The prediction of mobile genes was determined using the method of Duranti et al. (2017). Genes flanking the potential resistance-correlated mutant genes were analyzed in the range of $10 \mathrm{~kb}$ by using homology searches (Lugli et al., 2016) and Colombo software (Waack et al., 2006). Mobile elements, including conjugative transposons, episomes, and prophages, were predicted by homology searches against the database, which can be downloaded (http://probiogenomics.unipr.it/sw/ MobElemDB.zip) from the Probiogenomics Laboratory, University of Parma (Duranti et al., 2017).

\section{Statistical Analyses}

Minimum inhibitory concentrations were determined for 3 replicates, and the results were consistent between replicates. Replicates were only used to ensure no contamination or operator error had occurred. Figures presented in this paper were plotted using the software GraphPad Prism 5 (GraphPad Inc., La Jolla, CA).

\section{RESULTS}

\section{Experimental Evolution of Streptomycin Resistance}

Two L. plantarum ATCC14917 lineages evolved in parallel under increasing streptomycin concentration, revealing adaptation; that is, the development of resistance through experimental evolution. At the initiation of passaging, the $\mathrm{MIC}$ and $\mathrm{IC}_{50}$ of $L$. plantarum ATCC14917 to streptomycin were 16 and $8 \mu \mathrm{g} / \mathrm{mL}$, respectively. Cells of the initial isolate were exposed to $8 \mu \mathrm{g} / \mathrm{mL}$ streptomycin, and then the concentration was increased by 1.5 times every $5 \mathrm{~d}$. Over the course of passage, the MIC values of $L$. plantarum ATCC14917 increased significantly (Figure 1 ). The 2 replicate populations were propagated for $25 \mathrm{~d}$, at which time the concentration of streptomycin in MIC determination of streptomycin had reached its upper solubility limit

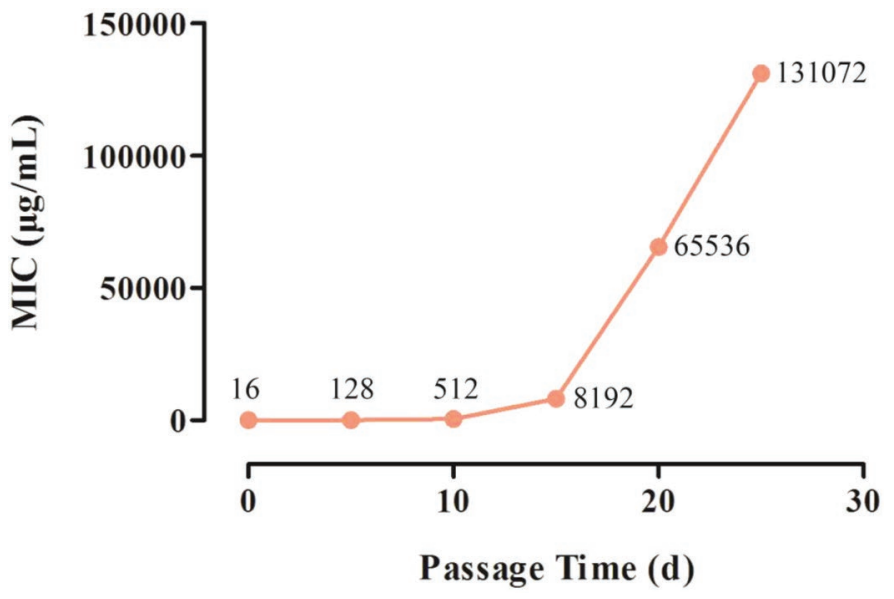

Figure 1. Changes in resistance (MIC) of Lactobacillus plantarum ATCC14917 to streptomycin during passage over a 25-d period. Color version available online.

$(0.1 \mathrm{~g} / \mathrm{mL})$. After $25 \mathrm{~d}$, the MIC value of L. plantarum ATCC14917 reached 131,072 $\mu \mathrm{g} / \mathrm{mL}$, which was 8,192-fold higher than that of the original parental isolate. For the control isolate, MIC remained constant throughout passage.

\section{Evolution of Resistant L. plantarum in the Absence of Antibiotic Pressure}

The L. plantarum ATCC14917 lineages with highlevel resistance that we obtained were then passaged in the absence of streptomycin selection pressure to investigate the stability of antibiotic resistance in both lineages. During passage in LSM without streptomycin, we detected a rapid decline in resistance (as measured by MIC). Although the MIC values decreased significantly, they stabilized at $2,048 \mu \mathrm{g} / \mathrm{mL}$ (Figure 2), remaining 128-fold higher than the MIC of the original isolate. These results showed that the development of resistance to streptomycin in L. plantarum ATCC14917 was an irreversible process; although MIC values declined in the absence of selection pressure, they stabilized at a level much higher than in the original isolate.

\section{Whole-Genome Sequencing and Validation of Mutated Genes}

The lineages were selected for whole-genome sequencing using the Illumina HiSeq 4000 platform: 2 original parent isolates, 2 control isolates, 2 evolved isolates following $25 \mathrm{~d}$ in a streptomycin environment, and 2 evolved high-resistance isolates following $35 \mathrm{~d}$ in a streptomycin-free medium. The matching rates between the obtained sequencing data and the reference genome were $>97.4 \%$. The mutations were established 


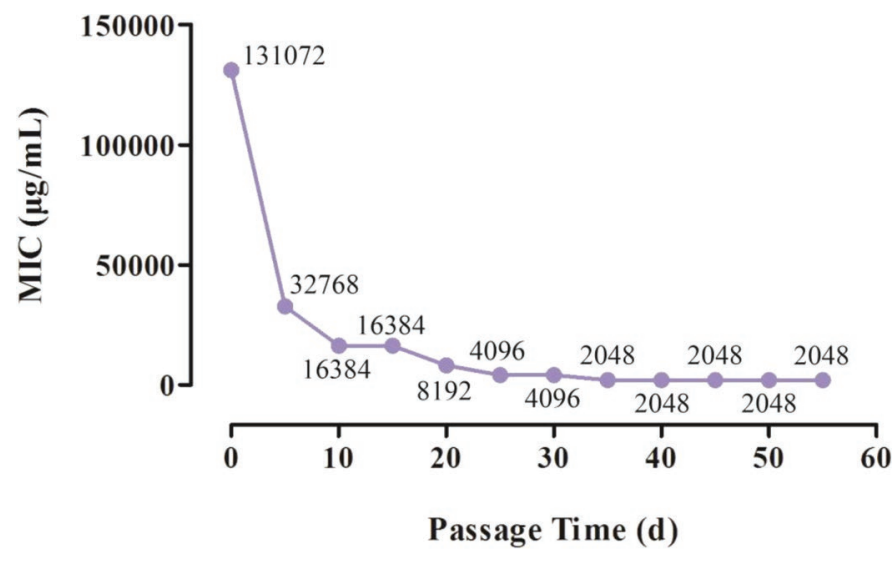

Figure 2. Changes in resistance (MIC) of Lactobacillus plantarum ATCC14917 in the absence of selection pressure. Color version available online.

for Track List (CLC Genomics Workbench, Qiagen), including matched sequence information, mutation information, reference genomic sequence, and coding sequence annotation, which were used for analysis of each mutation. Compared with the control and ancestor lineages, we identified 5 mutated genes, including 3 SNP or multi-nucleotide variants (MNV; Table 1) and 2 $\mathrm{SV}$ in L. plantarum ATCC14917 evolved in streptomycin, which were confirmed after validation using Sanger sequencing. The KEGG database, as an encyclopedia of genes and genomes, is widely used for analyzing not only genomics data but also transcriptomics, proteomics, glycomics, metabolomics, metagenomics, and other high-throughput data (Kanehisa et al., 2017). In this research, the functional categories of the metabolic pathways associated with mutations during evolution were annotated by the KEGG database. One mutated gene was annotated as ribosomal protein S12 (K02950) in an L. plantarum ATCC14917 isolate with high-level streptomycin resistance.

\section{Potential Mobility of Streptomycin Resistance- Related Mutant Genes in Highly Resistant L. plantarum ATCC14917 Lineages}

We performed homology-based analyses of the surrounding DNA regions of the mutant genes of $L$. plantarum ATCC14917 and showed that there were no mutant genes located in mobile elements (conjugative transposons, episomes, or prophages) in the genomic regions, indicating that horizontal transfer would not occur in these mutated genes.

\section{DISCUSSION}

In this research, we used a laboratory evolution method to study the adaptation of L. plantarum ATCC14917

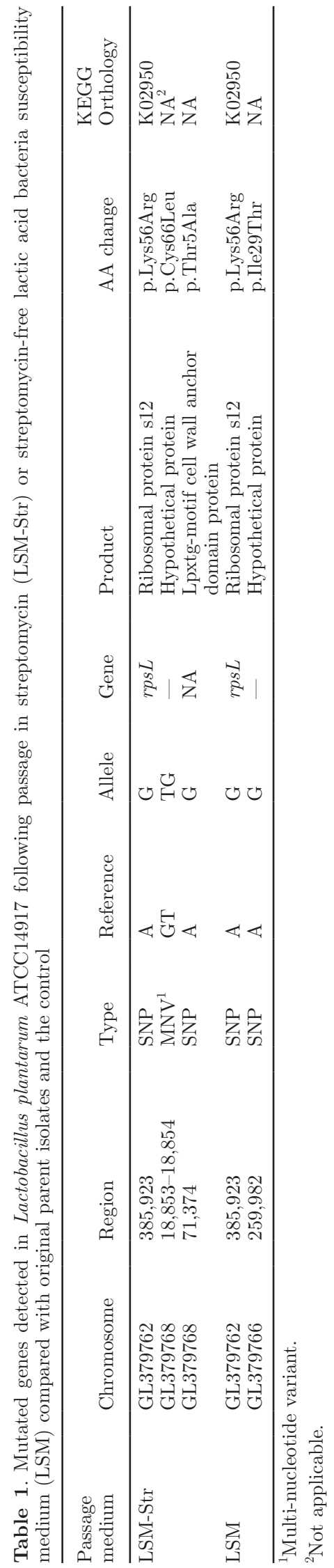

Journal of Dairy Science Vol. 101 No. 4, 2018 
to survival under stress in an environment containing streptomycin. Experimental evolution is an effective way to evaluate evolution in bacteria under different selection pressures. Tenaillon et al. (2016) sequenced a series of Escherichia coli lineages from one population over 50,000 generations and demonstrated the mutation and fitness trajectories of genome evolution; that research has become the reference study for using the laboratory evolution method to study adaptation in bacteria. For antibiotic resistance adaptation, Cowen et al. (2000) exposed Candida albicans to the antifungal agent fluconazole and passaged it over 330 generations under constant selection pressure. The final MIC value achieved in 2 populations was $64 \mu \mathrm{g} / \mathrm{mL}$, which was the highest level of fluconazole resistance ever observed in these isolates; the MIC remained constant during longer passage. In our previous work (Shao et al., 2015; Wang et al., 2017), adaptation in Lactobacillus casei Zhang was studied in LSM containing a constant concentration of amoxicillin or gentamicin during serial passage every $24 \mathrm{~h}$ for 2,000 generations; the results showed final MIC values of 8 and $32 \mu \mathrm{g} / \mathrm{mL}$ for amoxicillin and gentamicin, respectively, which was 4 and 16 times greater than that of the original parental isolate. We noted that maintaining a constant antibiotic concentration during the whole subculture process did not result in a remarkable increase in MIC. To study high-level resistance, we applied the experimental evolution technique with gradually increasing concentrations of the antibiotic (1.5 times higher than the previous dosage) at every fifth transfer and, in this way, obtained much higher levels of resistance.

With increasing streptomycin concentration during passage, the MIC values for L. plantarum ATCC14917 increased to $131,072 \mu \mathrm{g} / \mathrm{mL}$, which was 8,192-fold higher than the MIC of the original parent isolate. Subculturing was halted because the streptomycin concentration reached its upper solubility $(0.1 \mathrm{~g} / \mathrm{mL})$. Compared with using constant antibiotic concentrations, the gradually increased selection pressure was much more effective at achieving highly resistant isolates quickly. Toprak et al. (2011) developed a selection device that could continuously monitor the growth of Escherichia coli and dynamically adjust antibiotic concentration according to the actual rate at which resistance was evolving; at the end of the evolution experiments, resistance levels for chloramphenicol, doxycycline, and trimethoprim had increased by 870-, 10-, and 1,680-fold, respectively. Lázár et al. (2014) conducted high-throughput laboratory evolutionary experiments using $E$. coli K12, which was exposed to gradually increasing concentrations of antibiotics, and the results showed that isolates evolved successively higher levels of antibiotic resistance compared with parental isolates.
To further study the adaptation of resistance mechanisms in the evolved isolates, we used whole-genome sequencing to identify the genetic mutations (SNP, InDel, and SV) in 8 isolates of L. plantarum ATCC14917; namely, 2 original isolates, 2 control isolates, 2 isolates after $25 \mathrm{~d}$ in a streptomycin environment, and 2 highly resistant isolates after $35 \mathrm{~d}$ in streptomycin-free medium. The bioinformatics analysis results showed 3 SNP and $2 \mathrm{SV}$ in L. plantarum ATCC14917 that had evolved with streptomycin. We found that 1 SNP in the gene encoding small subunit ribosomal protein 12 (S12) might correlate with streptomycin resistance in ATCC14917, which needs to be confirmed by further study. Streptomycin acts by binding to the highly conserved A-site of the 16S rRNA 30S ribosomal subunit, inhibiting bacterial protein synthesis; S12 is the main site of action for streptomycin in the $30 \mathrm{~S}$ ribosomal subunit, and previous research has demonstrated that streptomycin resistance was associated with mutations responsible for ribosomal resistance (Finken et al., 1993). The normal role of the S12 protein is to combine with streptomycin, leading to misreading of the genetic code during translation, interfering with protein synthesis and resulting in subsequent bacterial death. A high level of streptomycin resistance is often linked to mutations within $r r s$, a $16 \mathrm{~S}$ rRNA gene, or rpsL, which encodes the ribosomal protein S12 (Finken et al., 1993; Honoré et al., 1995). A large number of studies have shown that mutation of the S12 ribosomal protein may change the ribosomal target site and confer bacteria with a significant level of resistance to streptomycin (Carter et al., 2000; Springer et al., 2001; Ogle and Ramakrishnan, 2005).

We also found SV in the $\operatorname{lrg} A$ gene, which may be involved in controlling cell death and lysis. Ranjit et al. (2011) studied the biochemical and molecular characterization of a novel family of cell-death effectors, of which the Lrg proteins were prototypical members; they confirmed that $\operatorname{LrgA}$ proteins are bacterial antiholin-like proteins that control cell death and lysis during biofilm development. Based on secondary structure similarities to bacteriophage-encoded antiholins and the phenotypes of $\operatorname{lrg}$ mutants, $\operatorname{lrg} A$ was proposed to encode an antiholin-like protein with an inhibitory effect on these enzymes (Groicher et al., 2000; Rice et al., 2003). Studies have shown that LrgA was also involved in the control of murein hydrolase activity. Zymographic and quantitative analysis of murein hydrolase activity revealed that the $\operatorname{lrg} A$ mutant had increased extracellular murein hydrolase activity compared with that of the wild-type isolate. In addition, the influence of the $\operatorname{lrg} A$ mutation on penicillin-induced lysis and death was determined (Groicher et al., 2000), indicating that mutations in the $\operatorname{lrg} A$ gene may inhibit 
extracellular murein hydrolase activity and encourage penicillin tolerance. Similarly, we found that when $L$. plantarum ATCC14917 was exposed to streptomycin, the mutation in $\operatorname{lrg} A$ murein might inhibit hydrolase activity and autolysis and strengthen streptomycin resistance.

Mutations in the serine/threonine phosphatase family protein were also detected in highly resistant $L$. plantarum ATCC14917 lineages and could be linked to streptomycin resistance. Serine/threonine protein phosphatase could control the level of phosphorylation of several proteins, and the enhancement of protein phosphorylation could activate L,D-transpeptidases, which in turn could cause peptidoglycan cross-linking by tetrapeptides, ultimately promoting the polymerization of peptide-synthetizing peptidoglycan, which is essential for normal growth and reproduction of bacteria (Sacco et al., 2014). Sacco et al. (2014) found that mutations in the serine/threonine protein phosphatase had a bearing on resistance to ampicillin in Enterococcus faecium.

One hypothetical protein-related gene mutation was found in the genome of L. plantarum ATCC14917 lineages with high resistance to streptomycin. This protein had an unverified function in GenBank and there was a lack of experimental evidence for the function of these proteins (Nan et al., 2009). However, this protein might be related to streptomycin resistance in L. plantarum ATCC14917. When the highly resistant isolates were passaged in the absence of streptomycin, the MIC of the lineages decreased by 128 -fold but then remained constant, indicating that resistance in these lineages was an irreversible process. By analyzing changes in the sequences of the mutated genes regulating the ribosomal protein S12, LPXTG-motif cell wall anchor domain protein, LrgA family protein, Ser/Thr phosphatase family protein, and a hypothetical protein in the evolved lineages when they were passaged in streptomycin-free medium, we found that only mutated genes regulating the ribosomal protein S12 could still be detected. This indicates that the ribosomal protein S12 may correlate with resistance of $L$. plantarum ATCC14917 to streptomycin, as demonstrated by the fact that the MIC value remained at $2,048 \mu \mathrm{g} / \mathrm{mL}$.

In this research, we also aimed to determine the likelihood of transfer of mutant genes from resistant L. plantarum ATCC14917 by locating any mobile elements. We demonstrated that the mutant genes responsible for streptomycin resistance were not located in mobile genetic elements such as conjugative transposons, episomes, or prophages. Combining probiotics with antibiotics to cure disease is a developing trend. Probiotics could help restore the gut microbiota disordered by antibiotics while enhancing immunity, promoting absorption of the nutrients, and regulating function of the gastrointestinal tract. However, the precondition for combined use of probiotics and antibiotics is that the probiotic must be resistant to the antibiotic and that the genes responsible for resistance must not be able to transfer to other organisms. Antibiotic databases such as the Antibiotic Resistance Genes Database (ARDB; Hu et al., 2013) and the Comprehensive Antibiotic Resistance Database (CARD; Jia et al., 2017) are available for prediction of antibiotic-resistant genes. Similarly, the mobile elements database of MobElemDB (http://probiogenomics.unipr.it/sw/MobElemDB.zip), NCBI RefSeq (https://www.ncbi.nlm.nih.gov/refseq/), INTEGRALL (http://integrall.bio.ua.pt/), and ISfinder (http://www-is.biotoul.fr/) are available to help predict resistance gene mobility (Guo et al., 2014).

Instead of studying gene transfer using in vivo or in vitro methods, whole-genome sequencing and bioinformatics analysis could be an effective way to comprehensively evaluate potential antibiotic-resistant genes and their mobility. In this study, we combined laboratory evolution studies and whole-genome sequencing to study resistant genes in L. plantarum ATCC14917 and preliminarily evaluate the safety of streptomycinresistant probiotics for potential use in combination with the antibiotic streptomycin.

\section{ACKNOWLEDGMENTS}

This research was supported by the Fundamental Research Funds for the Central Universities (grant nos. 2016CSY018, GK201603097, and GK201703063), the Major Transformation of Scientific and Technological Achievements Special Program of Shaanxi Province, China (no. 2016KTG01-02), the Science and Technology Research and Development Program of Shaanxi Province, China (no. 2016NY-207), and the Scientific Research Foundation of Hainan University (no. KYQD1548).

\section{REFERENCES}

Barrick, J. E., and R. E. Lenski. 2013. Genome dynamics during experimental evolution. Nat. Rev. Genet. 14:827-839.

Baym, M., T. D. Lieberman, E. D. Kelsic, R. Chait, R. Gross, I. Yelin, and R. Kishony. 2016. Spatiotemporal microbial evolution on antibiotic landscapes. Science 353:1147-1151.

Cairns, J., J. Frickel, M. Jalasvuori, T. Hiltunen, and L. Becks. 2017. Genomic evolution of bacterial populations under coselection by antibiotics and phage. Mol. Ecol. 26:1848-1859.

Carter, A. P., W. M. Clemons, D. E. Brodersen, R. J. Morganwarren, B. T. Wimberly, and V. Ramakrishnan. 2000. Functional insights from the structure of the $30 \mathrm{~S}$ ribosomal subunit and its interactions with antibiotics. Nature 407:340-308.

CLSI. 2006. Methods for antimicrobial dilution and disk susceptibility testing of infrequently isolated or fastidious bacteria; proposed guideline. M45-P. 25th ed. Clinical and Laboratory Standards Institute, Wayne, PA. 
Cowen, L. E., D. Sanglard, D. Calabrese, C. Sirjusingh, J. B. Anderson, and L. M. Kohn. 2000. Evolution of drug resistance in experimental populations of Candida albicans. J. Bacteriol. 182:1515-1522.

Duranti, S., G. A. Lugli, L. Mancabelli, F. Turroni, C. Milani, M. Mangifesta, C. Ferrario, R. Anzalone, A. Viappiani, D. Sinderen and M. Ventura. 2017. Prevalence of antibiotic resistance genes among human gut-derived bifidobacteria. Appl. Environ. Microbiol. 83:e02894-16.

European Food Safety Authority (EFSA). 2012. Guidance on the assessment of bacterial susceptibility to antimicrobials of human and veterinary importance. EFSA J. 10:2740.

Finken, M., P. Kirschner, A. Meier, A. Wrede, and E. C. Böttger. 1993. Molecular basis of streptomycin resistance in Mycobacterium tuberculosis: Alterations of the ribosomal protein S12 gene and point mutations within a functional $16 \mathrm{~S}$ ribosomal RNA pseudoknot. Mol. Microbiol. 9:1239-1246.

FAO-WHO. 2002. Joint FAO/WHO working group report on drafting guidelines for the evaluation of probiotics in food. Food and Agricultural Organization of the United Nations, Rome, Italy, and World Health Organization, Geneva, Switzerland.

Govender, M., Y. E. Choonara, S. van Vuuren, P. Kumar, L. C. du Toit, K. Erlwanger, and V. Pillay. 2016. A dual-biotic system for the concurrent delivery of antibiotics and probiotics: in vitro, ex vivo, in vivo and in silico evaluation and correlation. Pharm. Res. 33:3057-3071.

Groicher, K. H., B. A. Firek, D. F. Fujimoto, and K. W. Bayles. 2000. The Staphylococcus aureus $\operatorname{lrg} \mathrm{AB}$ operon modulates murein hydrolase activity and penicillin tolerance. J. Bacteriol. 182:1794-1801.

Guo, X., S. Liu, Z. Wang, X. Zhang, M. Li, and B. Wu. 2014. Metagenomic profiles and antibiotic resistance genes in gut microbiota of mice exposed to arsenic and iron. Chemosphere 112:1-8.

Honoré, N., G. Marchal, and S. T. Cole. 1995. Novel mutation in 16S rRNA associated with streptomycin dependence in Mycobacterium tuberculosis. Antimicrob. Agents Chemother. 39:769.

Hu, Y., X. Yang, J. Qin, N. Lu, G. Cheng, N. Wu, Y. Pan, J. Li, L. Zhu, X. Wang, Z. Meng, F. Zhao, D. Liu, J. Ma, N. Qin, C. Xiang, Y. Xiao, L. Li, H. Yang, J. Wang, R. Yang, G. F. Gao, J. Wang, and B. Zhu. 2013. Metagenome-wide analysis of antibiotic resistance genes in a large cohort of human gut microbiota. Nat. Commun. 4:2151.

ISO-IDF. 2010. Milk and milk products - Determination of the minimal inhibitory concentration (MIC) of antibiotics applicable to bifidobacteria and non-enterococcal lactic acid bacteria (LAB). IS010932/IDF223. International Organization for Standardization, Geneva, Switzerland, and International Dairy Federation, Brussels, Belgium.

Jia, B., A. R. Raphenya, B. Alcock, N. Waglechner, P. Guo, K. K. Tsang, B. A. Lago, B. M. Dave, S. Pereira, A. N. Sharma, S. Doshi, M. Courtot, R. Lo, L. E. Williams, J. G. Frye, T. Elsayegh, D. Sardar, E. L. Westman, A. C. Pawlowski, T. A. Johnson, F. S. Brinkman, G. D. Wright, and A. G. McArthur. 2017. CARD 2017: Expansion and model-centric curation of the comprehensive antibiotic resistance database. Nucleic Acids Res. 45:D566-D573.

Kanehisa, M., M. Furumichi, M. Tanabe, Y. Sato, and K. Morishima. 2017. KEGG: New perspectives on genomes, pathways, diseases and drugs. Nucleic Acids Res. 45:D353-D361.

Klare, I., C. Konstabel, S. Müller-Bertling, R. Reissbrodt, G. Huys, M Vancanneyt, J. Swings, H. Goossens, and W. Witte. 2005. Evaluation of new broth media for microdilution antibiotic susceptibility testing of lactobacilli, pediococci, lactococci, and bifidobacteria. Appl. Environ. Microbiol. 71:8982-8986.

Laxminarayan, R., D. Sridhar, M. Blaser, M. Wang, and M. Woolhouse. 2016. Achieving global targets for antimicrobial resistance. Science 353:874-875

Lázár, V., I. Nagy, R. Spohn, B. Csörgő, Á. Györkei, Á. Nyerges, B. Horváth, A. Vörös, R. Busafekete, and M. Hrtyan. 2014. Genomewide analysis captures the determinants of the antibiotic crossresistance interaction network. Nat. Commun. 5:4352.
Lenski, R. E. 2017. Experimental evolution and the dynamics of adaptation and genome evolution in microbial populations. ISME J. 11:2181-2194.

Levin-Reisman, I., I. Ronin, O. Gefen, I. Braniss, N. Shoresh, and N. Q. Balaban. 2017. Antibiotic tolerance facilitates the evolution of resistance. Science 355:826-830.

Lugli, G. A., C. Milani, L. Mancabelli, D. van Sinderen, and M. Ventura. 2016. MEGAnnotator: A user-friendly pipeline for microbial genomes assembly and annotation. FEMS Microbiol. Lett. 363:fnw049. https://doi.org/10.1093/femsle/fnw049.

Lukačišinová, M., and T. Bollenbach. 2017. Toward a quantitative understanding of antibiotic resistance evolution. Curr. Opin. Biotechnol. 46:90-97.

Monahan, J. C. 2011. The FDA and generally recognized as safe (GRAS) substances. Nova Science Publishers, Hauppauge, NY.

Nan, J., E. Brostromer, X. Y. Liu, O. Kristensen, and X. D. Su. 2009 Bioinformatics and structural characterization of a hypothetical protein from Streptococcus mutans: Implication of antibiotic resistance. PLoS One 4:e7245.

Ogle, J. M., and V. Ramakrishnan. 2005. Structural insights into translational fidelity. Annu. Rev. Biochem. 74:129-177.

Ranjit, D. K., J. L. Endres, and K. W. Bayles. 2011. Staphylococcus aureus CidA and LrgA proteins exhibit holin-like properties. J. Bacteriol. 193:2468-2476.

Rice, K. C., J. B. Firek, B. A. Nelson, S. J. Yang, T. G. Patton, and K. W. Bayles. 2003. The Staphylococcus aureus cidAB operon: Evaluation of its role in regulation of murein hydrolase activity and penicillin tolerance. J. Bacteriol. 185:2635-2643.

Sacco, E., M. Cortes, N. Josseaume, L. B. Rice, J. L. Mainardi, and M. Arthur. 2014. Serine/threonine protein phosphatase-mediated control of the peptidoglycan cross-linking L, D-transpeptidase pathway in Enterococcus faecium. MBio 5:e01446-e14.

Sánchez, B., S. Delgado, A. Blanco-Míguez, A. Lourenço, M. Gueimonde, and A. Margolles. 2017. Probiotics, gut microbiota, and their influence on host health and disease. Mol. Nutr. Food Res. 61. https://doi.org/10.1002/mnfr.201600240.

Shao, Y., W. Zhang, H. Guo, L. Pan, H. Zhang, and T. Sun. 2015. Comparative studies on antibiotic resistance in Lactobacillus casei and Lactobacillus plantarum. Food Control 50:250-258.

Springer, B., Y. G. Kidan, T. Prammananan, K. Ellrott, E. C. Böttger, and P. Sander. 2001. Mechanisms of streptomycin resistance: Selection of mutations in the 16S rRNA gene conferring resistance. Antimicrob. Agents Chemother. 45:2877-2884.

Stefanovic, E., G. Fitzgerald, and O. McAuliffe. 2017. Advances in the genomics and metabolomics of dairy lactobacilli: A review. Food Microbiol. 61:33-49.

Tenaillon, O., J. E. Barrick, N. Ribeck, D. E. Deatherage, J. L. Blanchard, A. Dasgupta, G. C. Wu, S. Wielgoss, S. Cruveiller, C Médigue, D. Schneider, and R. E. Lenski. 2016. Tempo and mode of genome evolution in a 50,000-generation experiment. Nature $536: 165-170$.

Tomaro-Duchesneau, C., M. L. Jones, D. Shah, P. Jain, S. Saha, and S. Prakash. 2014. Cholesterol assimilation by Lactobacillus probiotic bacteria: An in vitro investigation. BioMed Res. Int. 2014:380316.

Toprak, E., A. Veres, J. B. Michel, R. Chait, D. L. Hartl, and R. Kishony. 2011. Evolutionary paths to antibiotic resistance under dynamically sustained drug selection. Nat. Genet. 44:101.

Waack, S., O. Keller, R. Asper, T. Brodag, C. Damm, W. F. Fricke, K Surovcik, P. Meinicke, and R. Merkl. 2006. Score-based prediction of genomic islands in prokaryotic genomes using hidden Markov models. BMC Bioinform. 7:142.

Wang, J., X. Dong, Y. Shao, H. Guo, L. Pan, W. Hui, L. Y. Kwok, H. Zhang, and W. Zhang. 2017. Genome adaptive evolution of Lactobacillus casei under long-term antibiotic selection pressures. BMC Genomics 18:320.

Wang, P., Z. Wu, J. Wu, D. Pan, X. Zeng, and K. Cheng. 2016. Effects of salt stress on carbohydrate metabolism of Lactobacillus plantarum ATCC 14917. Curr. Microbiol. 73:491-497. 